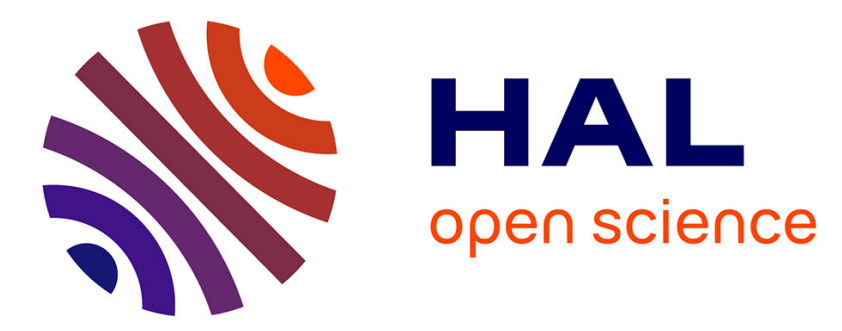

\title{
Poiseuille flow of nonlocal microstructured fluid
}

Arnaud Perrot, Noël Challamel, Vincent Picandet

\section{To cite this version:}

Arnaud Perrot, Noël Challamel, Vincent Picandet. Poiseuille flow of nonlocal microstructured fluid. Mechanics Research Communications, 2014, 59, pp.51-57. 10.1016/j.mechrescom.2014.04.008 . hal01115540

\author{
HAL Id: hal-01115540 \\ https://hal.science/hal-01115540
}

Submitted on 3 Feb 2020

HAL is a multi-disciplinary open access archive for the deposit and dissemination of scientific research documents, whether they are published or not. The documents may come from teaching and research institutions in France or abroad, or from public or private research centers.
L'archive ouverte pluridisciplinaire HAL, est destinée au dépôt et à la diffusion de documents scientifiques de niveau recherche, publiés ou non, émanant des établissements d'enseignement et de recherche français ou étrangers, des laboratoires publics ou privés. 


\title{
Poiseuille flow of nonlocal microstructured fluid
}

\section{A. Perrot ${ }^{1}$, N. Challamel $^{1}$ and V. Picandet ${ }^{1}$}

${ }^{1}$ Université de Bretagne Sud, EA 4250

Laboratoire d'Ingénierie des MATériaux de Bretagne, (LIMATB)

F-56100 Lorient, France

Email : arnaud.perrot@univ-ubs.fr

\begin{abstract}
:
In this paper, an Eringen's type nonlocal flow law is developed to account for the microstructured effects in modelling the behaviour of heterogeneous fluids. The axisymmetrical Poiseuille flow of Newtonian and non-Newtonian fluids is studied using the nonlocal mechanics, and some original analytical results are obtained for this geometrical configuration. It is shown that the nonlocal Newtonian fluid exhibits a kind of pseudo plastic behaviour because a plug flow zone exists within the concept of nonlocal mechanics. Furthermore, the shear rate profile shows some nonlinearity for the nonlocal Newtonian fluid, a property which cannot be accounted for with the so-called local theory. Viscoplastic fluids, modelled by Herschel-Bulkley or Bingham laws, are also investigated using this nonlocal generalization. Hypergeometric functions are used to compute velocity profiles. A general parametric study illustrates the nonlocal specificities of the viscoplastic flow for such heterogeneous materials. Finally, the Newtonian nonlocal model is calibrated using a discrete layered axisymmetrical Poiseuille flow.
\end{abstract}

Keywords: Nonlocal ; Non-newtonian fluids ; Viscoplasticity ; Microstructured fluids ; Scale effect ; Poiseuille geometry ; Analytical solution ; Herschel-Bulkley law

\section{Introduction}

Recently, flow of concentrated suspensions or emulsions have shown finite size effects such as spatial cooperativity or localized plastic shear banding. According to Goyon et al. [1], there is a strong need to develop a theoretical framework to describe the non-local flow behaviour of glassy and jammed systems, such as soft glasses and granular materials. These authors suggested that nonlocal theory can be used to model these finite size effects.

Nonlocal mechanics is a continuum mechanics theory that may be able to capture some detailed phenomena ranging from small to large scale. Nonlocal mechanics was largely developed in the 60's notably for solid mechanics applications (see the historical perspective of Maugin [2]; see also the book of Eringen) [2,3]. Among the different nonlocal mechanics theories, integral-based nonlocal models have found a large diversity of applications, especially in the fields of heterogeneous, discrete solids and microcracking media where they are used to eliminate the stress singularities in the vicinity of a crack. The application of integral-based nonlocal mechanics to fluid mechanics was developed in the 70's, with the Eringen seminal paper on integral-based nonlocal Newtonian fluids [4]. Recently, with the interest in micro fluids and atomic fluids mechanics, nonlocal concepts have found new applications (see for instance Todd and Hansen [5] or Puscasu et al [6]). The nonlocal kernels or nonlocal viscosity can be calibrated using simple atomic fluids (see for instance Alley and Alder [7] or 
more recently Puscasu et al [6]). It is worth mentioning that some spatial related nonlocal concepts have also been introduced to explain the flow cooperativity phenomenon $[1,8,9]$. The nonlocal aspect of material parameters has been introduced by the previously referenced authors through a cooperativity length scale, which is linked to the microstructure geometry and used to compute a nonlocal viscosity parameter. A theoretical investigation of nonlocal fluids is also reported in the recent paper of Zhu et al [10].

In this study, a nonlocal Newtonian and non-Newtonian model is presented in a differential form. This model can be viewed as being analogous to the Eringen's model for solid mechanics (see the differential format of the elasticity stress-strain law of Eringen [11]) which has now been generalized to fluid materials. Axisymmetrical Poiseuille flow is studied using this nonlocal model, and some original analytical results are obtained for this geometrical configuration. The effect of the nonlocality on the modification of the viscoplastic fluid behaviour is also studied. Regarding Newtonian behaviour, the proposed model is compared to a discrete layered axisymmetrical Poiseuille flow and the nonlocal characteristic length is calibrated with the discrete layers thickness.

\section{Behaviour and flow geometry}

The uniaxial Herschel-Bulkley law is studied in a nonlocal form in order that some nonlocal effects can be introduced:

$$
\left\langle|\tau|-l_{c}^{2} \Delta|\tau|-\tau_{0}\right\rangle=\mu(|\dot{\gamma}|)^{n}
$$

where $\langle x\rangle=\frac{x+|x|}{2}$ is the positive operator. $\tau$ is the shear stress and $\dot{\gamma}$ is the shear rate. The length scale parameter $l_{c}$ controls the nonlocal aspect of the constitutive law. Eq. (1) can be also written as:

$$
\tau-l_{c}^{2} \Delta \tau-\tau_{0}=\mu \dot{\gamma}^{n} \text { for } \dot{\gamma} \geq 0 \text { and } \tau-l_{c}^{2} \Delta \tau+\tau_{0}=\mu \dot{\gamma}^{n} \text { for } \dot{\gamma} \leq 0
$$

The Herschel-Bulkley law covers many of the viscoplastic rheological laws including the particular case for given values of $\tau_{0}$ and $n$. For instance, the Bingham law is obtained for $n=1$; the power law is obtained for $\tau_{0}=0$ and the Newtonian law for $n=1$ and $\tau_{0}=0$. Eq. (1) can be considered as the Eringen's type nonlocal generalization of the Herschel-Bulkley law. This kind of Laplace operator affecting the stress variable was already suggested by Eringen [11] for elastic solids materials.

In this paper axisymmetric Poiseuille flow is considered only in the direction of the $\mathrm{x}$-axis and associated with a constant pressure gradient, denoted by $A>0$. With this geometrical configuration, if we assume that gravitational forces are negligible, the $\mathrm{x}$-axis term of the stress equilibrium can be written as $\mathrm{Eq}(3)$ :

$$
\frac{\partial \sigma_{x}}{\partial x}+\frac{1}{r} \frac{\partial\left(r \tau_{x r}\right)}{\partial r}=0
$$

The flow conditions dictates that $\sigma_{x}(x, r)=-p(x)$. Substituting $\tau$ for $\tau_{x r}$ the $\mathrm{x}$-axis term of the divergence of the stress tensor leads to Eq. (4).

$$
\frac{1}{r} \frac{d(r \tau)}{d r}=\frac{d p}{d x}=A
$$

Integration of equation (4) results in the relationship:

$$
\tau=\frac{A r}{2}+\frac{B}{r}
$$

where $B$ is a constant. The only possible value for B is zero to have a finite value of the shear stress along the radial axis.

\section{Shear rate profile and evolution of the plug flow zone}

For "local" viscoplastic materials, two cases can be distinguished. The first case occurs at low values of the pressure gradient if the shear stress does not exceed the yield stress in the gap; in this case, no flow occurs. In the second case, the material is sheared at a radius greater than a critical value $r_{c}$ where the shear stress is higher than the yield stress $\tau_{0}$. Thereafter for nonlocal materials, if $R \geq r_{c} \geq 0$ the flow is described by the nonlocal stress law: 
$\tau-l_{c}^{2} \Delta \tau-\tau_{0}=\mu \dot{\gamma}^{n}$

The following dimensionless parameters can be considered:

$r^{*}=\frac{r}{R}, l_{c}^{*}=\frac{l_{c}}{R}, \tau^{*}=\frac{2 \tau}{A R}=\frac{\tau}{\tau_{\max }}, \tau_{0}^{*}=\frac{2 \tau_{0}}{A R}=\frac{\tau_{0}}{\tau_{\max }}, \dot{\gamma}^{*}=\left(\frac{2 \mu}{A R}\right)^{1 / . n} \dot{\gamma}$

It is noted that the material is sheared if $\tau^{*}<1$. According to Eq. (5), the dimensionless shear stress can be written as:

$\tau^{*}\left(r^{*}\right)=r^{*}$

Consequently, we can write Eq. (6) in terms of a dimensionless radius:

$r^{*}-l_{c}^{* 2} \frac{1}{r^{*}} \frac{\partial}{\partial r^{*}}\left(r^{*} \frac{\partial\left(r^{*}\right)}{\partial r^{*}}\right)-\tau_{0}^{*}=\dot{\gamma}^{* n}$

the solution of which is given by:

$\dot{\gamma}^{* n}=r^{*}-\frac{l_{c}^{* 2}}{r^{*}}-\tau_{0}^{*}$

For Herschel-Bulkley and Bingham fluids, the plastic zone is defined by the position of the critical radius $r_{c}$ which is defined as being at the beginning of the sheared zone:

$\tau^{*}\left(r_{c}\right)=\tau_{0}^{*}$

Then, a dimensionless critical radius $r_{c}{ }^{*}=r_{c} / R$ can be introduced from the shear stress condition:

$\dot{\gamma}^{*}\left(r_{c}^{*}\right)=r_{c}^{*}-\frac{l_{c}^{* 2}}{r_{c}^{*}}-\tau_{0}^{*}=0 \Rightarrow r_{c}^{* 2}-\tau_{0}^{*} r_{c}^{*}-l_{c}^{* 2}=0, r_{c} \neq 0$

It should be noted that this plastic zone equation has a unique solution of $r_{c}{ }^{*}$ within the interval $] 0 ; 1[$ :

$r_{c}^{*}=\frac{\tau_{0}^{*}+\sqrt{\tau_{0}^{* 2}+4 l_{c}^{* 2}}}{2}$

The local case can be determined asymptotically as the length scale $1_{\mathrm{c}}{ }^{*}$ tends to zero, this being associated with the local critical radius $r_{c}^{*}=\tau_{0}^{*}$. Eq. (13) predicts a continuous increase of the dimensionless plug-flow radius $r_{c}{ }^{*}$ with respect to the characteristic length $l_{c}{ }^{*}$. The Newtonian and Power law fluids can be seen as a particular case of Herschell-Bulkley fluids with $\tau_{0}=0$. Consequently, for nonlocal viscous fluid, Eq. (13) leads to $r_{c}{ }^{*}=l_{c}{ }^{*}$. It follows that the nonlocal viscous fluid has a plug flow zone of radius $l_{c}{ }^{*}$ within a Poiseuille geometry. This means that the material is not sheared inside a zone controlled by the characteristic length scale which is linked to the material's microstructure. From a physical point of view, this can be attributed to the fact that inside the nonlocal critical radius, interactions between inclusions induce a "pseudo-plastic" material behaviour leading to unsheared zones.

For Herschel-Bulkley fluids, the shear rate is given by Eq. (10):

$\begin{cases}\dot{\gamma}^{*}=\left[r^{*}-\frac{l_{c}^{* 2}}{r^{*}}-\tau_{0}^{*}\right]^{1 / n} & ; r^{*}>r_{c}^{*} \\ \dot{\gamma}^{*}=0 & ; r^{*}<r_{c}^{*}\end{cases}$

For a Bingham fluid, the shear rate profile is obtained from Eq. (14) by taking $n=1$. Shear rate profiles of the nonlocal Herschel-Bulkley fluids $\left(\tau_{0}{ }^{*}=0.25\right)$ for a shear-thinning behaviour $(n=0.5)$, for a shear-thickening behaviour $(n=1.5)$ and for Bingham fluids are plotted in Figure 1. 


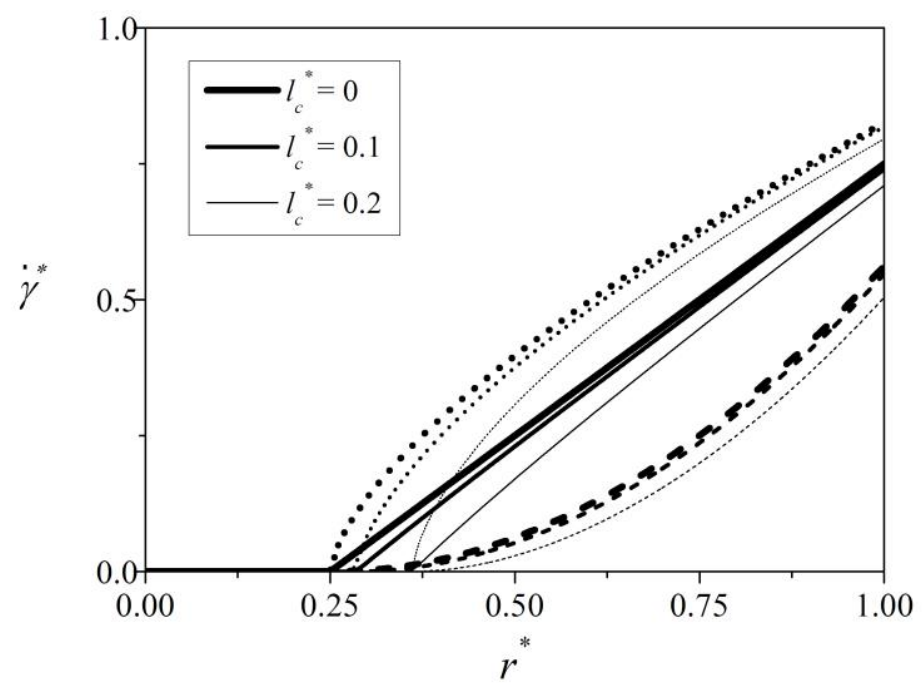

Figure 1: Shear rate profile for nonlocal viscoplastic fluids $\left(\tau_{0}{ }^{*}=0.25\right)$ with $l_{c}{ }^{*}$ ranging from 0 to 0.2 for Herschel-Bulkley fluid with $n=0.5$ (dashed lines), Herschel-Bulkley fluid with $n=1.5$ (dotted lines) and Bingham fluid (solid lines).

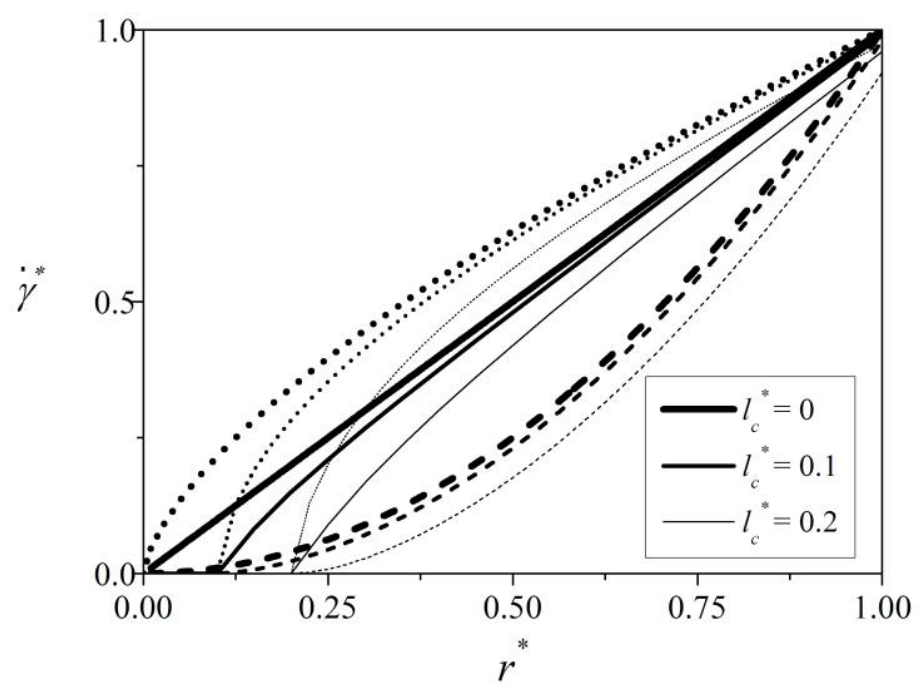

Figure 2: Shear rate profile for nonlocal viscous fluids $\left(\tau_{0}{ }^{*}=0.25\right)$ with $l_{c}{ }^{*}$ ranging from 0 to 0.2 for Power law fluid with $n=0.5$ (dashed lines), Power law fluid with $n=1.5$ (dotted lines) and Newtonian fluid (solid lines).

It is worth noting that nonlocal effects appear to strengthened the material, regardless of the flow index $n$, because the apparent viscosity of the material (i.e. $\tau / \dot{\gamma}$ ) increases with respect to the nonlocal length scale. The model also predicts that the size of the plug flow zone increases with the characteristic length. This is in agreement with experimental observation of Goyon et al. on the Poiseuille flow of emulsions exhibiting nonlocal finite size effects during flow [8]. Moreover, it can be noted that the linear shear rate profile obtained for the local Bingham behaviour disappears when nonlocal effects are introduced into the constitutive law.

In the case of a power law fluid, the shear rate can be obtained from Eq. (14) by taking $\tau_{0}{ }^{*}=0$ and for Newtonian fluid, $n=1$ and $\tau_{0}{ }^{*}=0$. Shear rate profiles for nonlocal viscous fluids are plotted in Figure 2 for a power law shear-thinning behaviour $(n=0.5)$, a power law shear-thickening behaviour $(n=$ 1.5) and a Newtonian fluid. The same apparent viscosity increase (of the nonlocal fluid), obtained for viscoplastic fluids can be seen in figure 2 for viscous fluids. Similarly, in the case of Bingham 
materials, the linearity of the shear rate profile obtained for the local Newtonian behaviour is lost when nonlocal effects are introduced in the constitutive law.

\section{Velocity Profile and flow rate}

\subsection{Velocity profile}

Dimensionless velocity can be defined as $V^{*}=\frac{1}{R}\left(\frac{2 \mu}{A R}\right)^{1 / n} V_{x}$.

For Herschel-Bulkley fluids and for $r^{*}>r_{c}{ }^{*}$ the non-dimensional velocity can be obtained by integrating the shear rate profile Eq. (14). To perform the spatial integration, Eq. (14) can be factorized as follows:

$$
\dot{\gamma}^{*}=r^{1 / n}\left(1^{*}-\frac{\tau_{0}^{*}+\sqrt{\tau_{0}^{* 2}+4 l_{c}^{* 2}}}{2 r^{*}}\right)^{1 / n}\left(1^{*}-\frac{\tau_{0}^{*}-\sqrt{\tau_{0}^{* 2}+4 l_{c}^{* 2}}}{2 r^{*}}\right)^{1 / n}
$$

which can be expanded in Taylor series:

$$
\dot{\gamma}^{*}=r^{1 / n} \sum_{k=0}^{\infty} \sum_{l=0}^{\infty} \frac{(-1 / n)_{k}(-1 / n)_{l}\left(\frac{\tau_{0}^{*}+\sqrt{\tau_{0}^{* 2}+4 l_{c}^{* 2}}}{2 r^{*}}\right)^{k}\left(\frac{\tau_{0}^{*}+\sqrt{\tau_{0}^{* 2}+4 l_{c}^{* 2}}}{2 r^{*}}\right)^{l}}{k ! l !}
$$

One can recognize that the double series is equal to a hypergeometric function of two variables $F_{l}$ as defined by Appell [12]:

$$
F_{1}\left(a ; b_{1}, b_{2} ; c ; z_{1}, z_{2}\right)=\sum_{k=0}^{\infty} \sum_{l=0}^{\infty} \frac{(a)_{k+l}\left(b_{1}\right)_{k}\left(b_{2}\right)_{l} z_{1}^{k} z_{2}^{l}}{(c)_{k+l} k ! l !} ;\left|z_{1}\right|<1 \wedge\left|z_{2}\right|<1
$$

where the rising Pochhammer symbol $(q)_{k}$ is used in the double series, and is defined by $(q)_{k}=q(q+1) \ldots(q+n-1)$ if $k>0$ and $(q)_{k}=1$ if $k=0$.

Then, according to Appell, Eq. (16) can be written as follow:

$$
\dot{\gamma}^{*}=r^{1 / n} F_{1}\left[1 ;-\frac{1}{n},-\frac{1}{n} ; 1 ; \frac{\tau_{0}^{*}+\sqrt{\tau_{0}^{* 2}+4 l_{c}^{* 2}}}{2 r^{*}}, \frac{\tau_{0}^{*}-\sqrt{\tau_{0}^{* 2}+4 l_{c}^{* 2}}}{2 r^{*}}\right]
$$

For the particular case of $F_{1}$ with $a=c$ and $b_{1}=b_{2}=b$, the integration rule of the Appell function is as follows:

$$
\int z^{1 / m} F_{l}\left(a ; b, b ; a ; r_{1} / z, r_{2} / z\right) d z=\frac{m}{m+1} z^{1+1 / m} F_{1}\left(\frac{-1+m}{m} ; b, b ;-1 / m ; r_{1} / z, r_{2} / z\right)
$$

This integration rule leads to the following equation for the velocity profile:

$$
V^{*}(r)=\frac{n}{n+1} r^{\frac{n+1}{n}} F_{1}\left[\frac{n-1}{n} ;-\frac{1}{n},-\frac{1}{n} ;-\frac{1}{n} ; \frac{\tau_{0}^{*}+\sqrt{\tau_{0}^{* 2}+4 l_{c}^{* 2}}}{2 r^{*}}, \frac{\tau_{0}^{*}-\sqrt{\tau_{0}^{* 2}+4 l_{c}^{* 2}}}{2 r^{*}}\right]+V_{1}^{*}
$$

with $V_{l}^{*}$, a constant defined to suit the no-slip boundary condition $V^{*}(1)=0$.

For Bingham fluids $(n=1)$, the integration of the shear rate profile lead to a simpler relationship. If 1 $\geq r^{*}>r_{c}{ }^{*}$, the velocity profile can be obtained by integrating the shear rate profile of a Bingham fluid knowing that $V_{x}^{*}(1)=0$ :

$$
V^{*}\left(r^{*}\right)=\frac{r^{* 2}-1}{2}-l_{c}^{* 2} \ln \left(r^{*}\right)+\tau_{0}^{*}\left(1-r^{*}\right)
$$

In the case of $r^{*}<r_{c}{ }^{*}$, velocity continuity can be used to obtain the entire velocity profile:

$$
V^{*}\left(r^{*}<r_{c}^{*}\right)=-\frac{1}{2}\left(-\frac{\tau_{0}^{*}}{2}\left[\tau_{0}^{*}+\sqrt{\tau_{0}^{* 2}+4 l_{c}^{* 2}}-4\right]+l_{c}^{* 2}-1-2 l_{c}^{* 2} \ln \left(\frac{\tau_{0}^{*}+\sqrt{\tau_{0}^{* 2}+4 l_{c}^{* 2}}}{2}\right)\right)
$$


Velocity profiles for nonlocal viscoplastic fluids $\left(\tau_{0}{ }^{*}=0.25\right)$ are plotted in Figure 3 for a shearthinning Herschel-Bulkley behaviour $(n=0.5)$, a shear-thickening Herschel-Bulkley behaviour $(n=$ 1.5) and Bingham behaviour. It appears that the velocity values decrease with respect to the nonlocal length scale for all dimensionless radii.

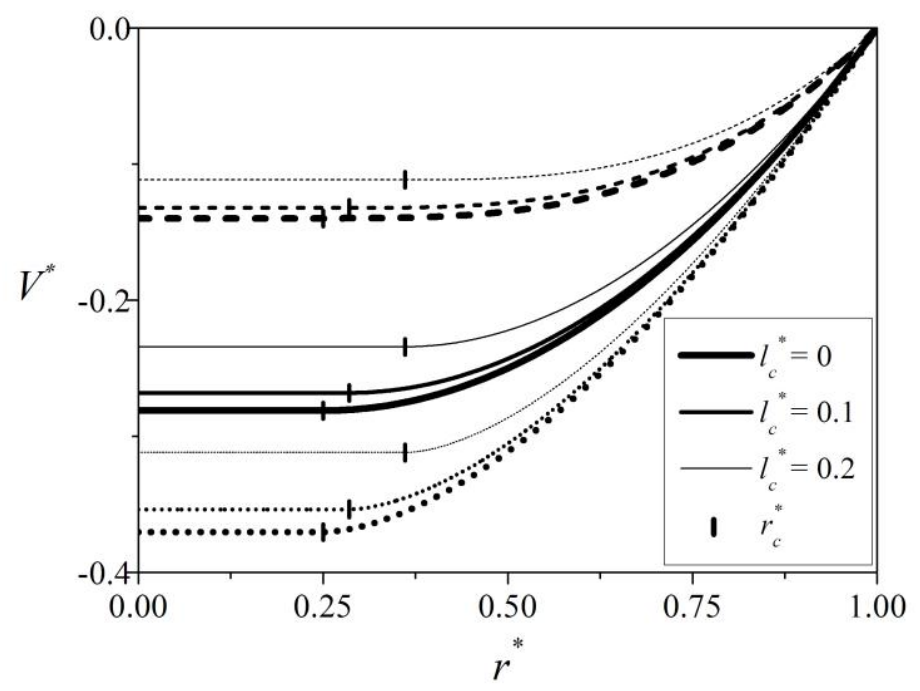

Figure 3: Velocity profile for nonlocal viscoplastic fluids $\left(\tau_{0}{ }^{*}=0.25\right)$ with $l_{c}{ }^{*}$ ranging from 0 to 0.2 for Herschel-Bulkley fluid with $n=0.5$ (dashed lines), Herschel-Bulkley fluid with $n=1.5$ (dotted lines) and Bingham fluid (solid lines).

If $\tau_{0}=0$ (i.e. for a power law fluid), the velocity profile can be obtained, when $r^{*}>l_{c}{ }^{*}$, by the integration of Eq. (14) with:

$V^{*}\left(r^{*}\right)=\frac{r^{* 1 / n+1}}{1 / n+1^{2}} F_{l}\left(-\frac{1 / n+1}{2} ;-1 / n ; \frac{-1 / n+1}{2} ; \frac{l_{c}^{* 2}}{r^{* 2}}\right)+V_{2}$

where $V_{2}$ is a constant defined with $V^{*}(1)=0$ and ${ }_{2} F_{1}$ the Gauss hypergeometric function defined as follow [13] for $|z|<1$ :

${ }_{2} F_{1}(a ; b ; c ; z)=\sum_{l=0}^{\infty} \frac{(a)_{k}(b)_{k} z^{k}}{(c)_{k} k !}$

This integration result can be proven using the basic properties of hypergeometric functions. Firstly, the shear rate profile equation is factorized by $r^{*}$ as follows:

$\dot{\gamma}^{*}=r^{1 / n}\left(1^{*}-\frac{l_{c}^{* 2}}{r^{2^{*}}}\right)^{1 / n}$

It is interesting to note that the Taylor series of the function $\left(1-z^{2}\right)^{b}$ is equal to the following hypergeometric function:

$\left(1-z^{2}\right)^{b}={ }_{2} F_{1}\left(-n ; a ; a ; z^{2}\right)$

where $a, b$ and $n$ are non zero real numbers. According to Eq. (26), Eq. (25) can be expressed as:

$\dot{\gamma}^{*}=r^{1 / n}{ }_{2} F_{l}\left(-\frac{1}{n}, 1,1, \frac{l_{c}^{* 2}}{r^{* 2}}\right)$

It is possible to perform a change of variable based on $x=l_{c}{ }^{*} / r^{*}$.

$\dot{\gamma}^{*}=\frac{l_{c}^{* 1 / n}}{x^{1 / n}} F_{1}\left(-\frac{1}{n}, 1,1, x^{2}\right)$

The velocity profile can be obtained by integrating the shear rate profile between $x=l_{c}{ }^{*}$ and $x=1$. 


$$
V^{*}\left(r^{*}\right)=\int_{l_{c}^{*}}^{1} \frac{l_{c}^{* 1 / n}}{x^{1 / n}}{ }_{2} F_{l}\left(-\frac{1}{n}, 1,1, x^{2}\right) \frac{l_{c}^{*} d x}{x^{2}}=\int_{l_{c}^{*}}^{1} \frac{l_{c}^{* 1 / n+1}}{x^{1 / n+2}}{ }_{2} F_{I}\left(-\frac{1}{n}, 1,1, x^{2}\right) d x
$$

the solution of which is given by the following integration rules of hypergeometric functions $[13,14]$ :

$$
\int z^{m}{ }_{2} F_{l}\left(a, b, b, z^{2}\right) d z=z_{2}^{1-m} F_{l}\left(\frac{1-m}{2}, a, 1+\frac{1-m}{2}, z^{2}\right)
$$

The resolution of equation (29) is then:

$$
V^{*}(x)=\frac{l_{c}^{* 1 / n+1}}{x^{1 / n+1}}{ }_{2} F_{1}\left(-\frac{1 / n+1}{2},-n,-\frac{1+n}{2}, x^{2}\right)+V_{2}
$$

Finally, for a Newtonian nonlocal fluid, the velocity profile is simply reduced to:

$$
\left\{\begin{array}{l}
V^{*}\left(r^{*}\right)=\frac{r^{* 2}-1}{2}-l_{c}^{* 2} \ln \left(r^{*}\right) ; r^{*}>l_{c}^{*} \\
V^{*}\left(r^{*}\right)=\frac{l_{c}^{* 2}-1}{2}-l_{c}^{* 2} \ln \left(l_{c}^{*}\right) ; r^{*}<l_{c}^{*}
\end{array}\right.
$$

Velocity profiles for nonlocal viscous fluids are plotted in Figure 4 for a shear-thinning behaviour $(n=$ $0.5)$, a shear-thickening behaviour $(n=1.5)$ and a Newtonian behaviour. It is interesting to note that when $r^{*}$ is less than $l_{c}^{*}$, the velocity is constant and equal to $V^{*}\left(l_{c}^{*}\right)$; the velocity profile then exhibits a plug flow zone exactly as found in the case of viscoplastic materials.

\subsection{Flow rate}

In the Poiseuille geometry, the flow rate is the key macroscopic parameter that is measured during the flow. A dimensionless mass flow rate is defined as follow:

$$
Q^{*}=-\left(\frac{2 \mu}{A R}\right)^{1 / n} \frac{1}{2 \pi R^{3}} Q
$$

The dimensionless flow rate can be linked to the dimensionless velocity by the following integral:

$$
Q^{*}=-\int_{0}^{1} V^{*}\left(r^{*}\right) r^{*} d r^{*}
$$

For viscoplastic materials (Bingham and Herschel-Bulkley), this equation can be further developed by taking into account the plug flow zone:

$$
Q^{*}=-V^{*}\left(r_{c}^{*}\right) \int_{0}^{r_{c}^{*}} r^{*} d r^{*}-\int_{r_{c}^{*}}^{1} V^{*}\left(r^{*}\right) r^{*} d r^{*}
$$

For nonlocal viscous materials, the plug flow zone is limited to a central zone limited by a radius equal to the nonlocal characteristic length $l_{c}{ }^{*}$. For Herschel-Bulkley fluids, a numerical integration is used to obtain the dimensionless flow rate as shown on Figure 5.

For Bingham fluids, the combination of Eq. (22), (23) and (35) yields the explicit expression of the mass flow rate:

$$
Q^{*}=-\frac{1}{8}\left[\left(\frac{\tau_{0}^{*}+\sqrt{\tau_{0}^{* 2}+4 l_{c}^{* 2}}}{2}\right)^{4}-1\right]+\frac{l_{c}^{* 2}}{4}\left[\left(\frac{\tau_{0}^{*}+\sqrt{\tau_{0}^{* 2}+4 l_{c}^{* 2}}}{2}\right)^{2}-1\right]+\frac{\tau_{0}^{*}}{6}\left[\left(\frac{\tau_{0}^{*}+\sqrt{\tau_{0}^{* 2}+4 l_{c}^{* 2}}}{2}\right)^{3}-1\right]
$$

The evolution of the dimensionless flow rate with respect to the characteristic length for viscoplastic materials is plotted on Figure 5. It clearly appears that the flow rate decreases with an increase in the characteristic length.

For Newtonian fluids, Eq. (35) provides the following dimensionless flow rate:

$Q^{*}=\frac{1}{8}\left[1-l_{c}^{* 4}\right]+\frac{l_{c}^{* 2}}{4}\left[l_{c}^{* 2}-1\right]$

The evolution of the dimensionless flow rate with respect to the characteristic length for viscous materials is plotted on Figure 5. As for nonlocal viscoplastic materials, it is evident that the flow rate decreases with increasing characteristic length. 


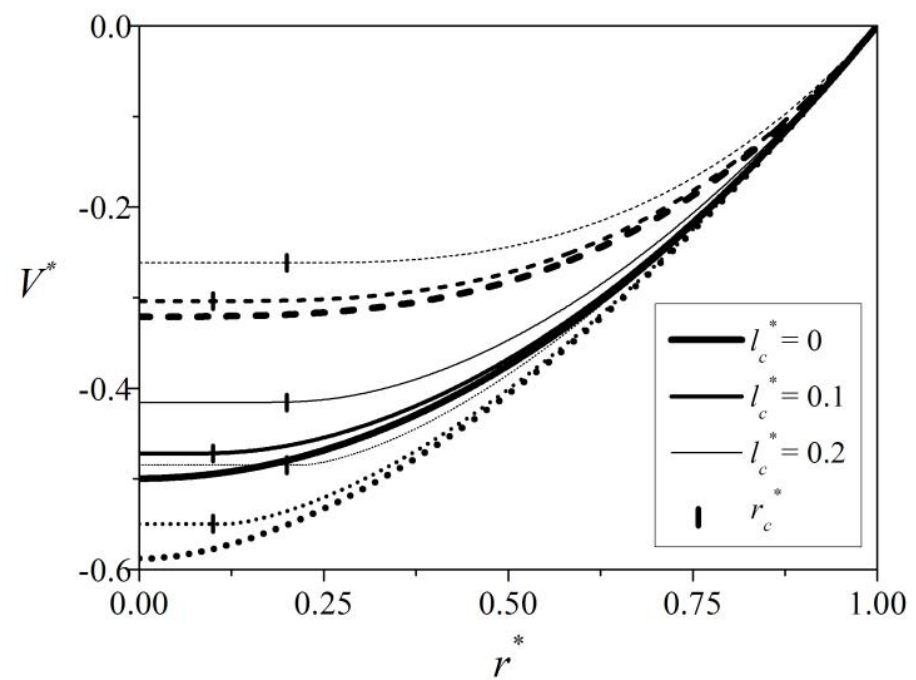

Figure 4: Velocity profile for nonlocal viscous fluids $\left(\tau_{0}{ }^{*}=0.25\right)$ with $l_{c}{ }^{*}$ ranging from 0 to 0.2 for Power law fluid with $n=0.5$ (dashed lines), Power law fluid with $n=1.5$ (dotted lines) and Newtonian fluid (solid lines).

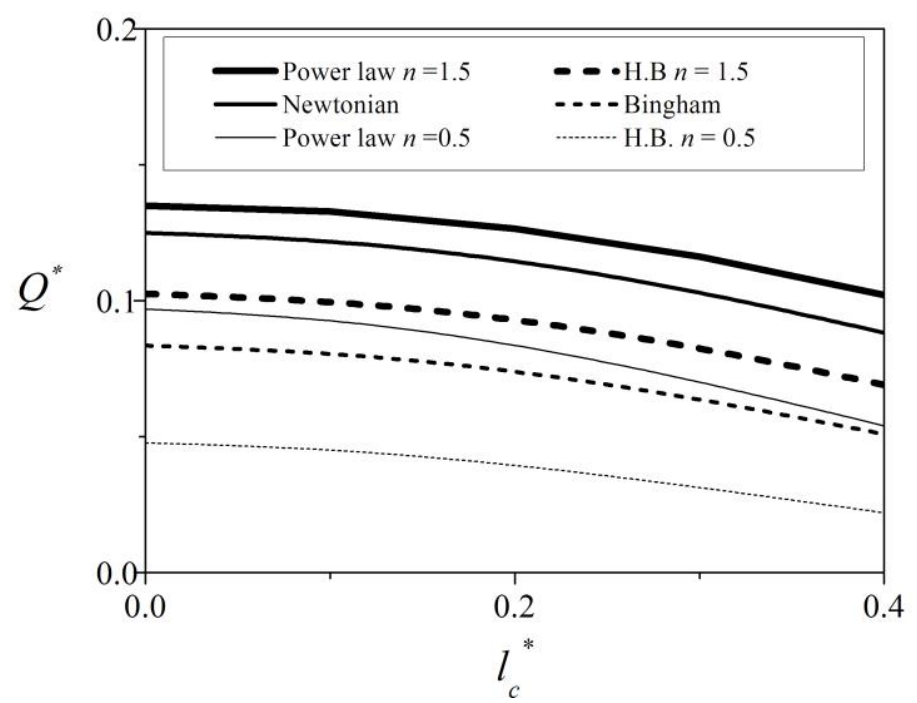

Figure 5: Dimensionless flow rate vs. dimensionless characteristic length for nonlocal viscous fluids (solid lines) and viscoplastic fluids with $\tau_{0}{ }^{*}=0.25$ (dashed lines) for $l_{c}{ }^{*}$ ranging from 0 to 0.4 (H.B. signifies Herschel-Bulkley).

\section{Calibration of the model with a discrete layered material for Newtonian behaviour}

\subsection{Discrete system: definition and velocity profile}

In order to give a physical basis to the nonlocal model, the nonlocal characteristic length is calibrated on the dimensionless layer thickness $a^{*}$ of a discrete concentric material flowing in a Poiseuille geometry. The calibration consists of finding the value of $l_{c}{ }^{*}$ that gives the best fit between the nonlocal and the discrete velocity profiles 


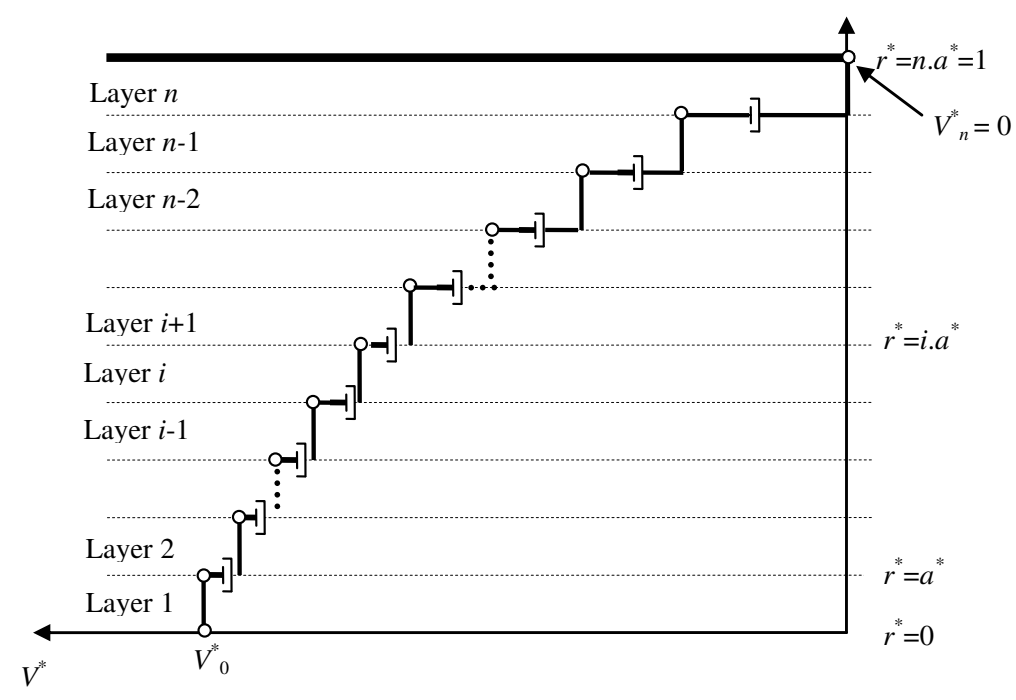

Figure 6: Studied discrete system consisting of $n$ concentric cylindrical layers of thickness $a$ separated by uniform viscous elements. The associate velocity profile is drawn, $a^{*}=1 / n$.

The stress equilibrium on an elemental layer provides the following discrete equation:

$2 \pi r_{i+1} \tau_{i+1}-2 \pi r_{i} \tau_{i}=A\left(\pi r_{i+1}^{2}-\pi r_{i}^{2}\right)$

which can be simplified in a dimensionless format:

$r_{i}^{*}+r_{i+1}^{*}=\frac{r_{i+1}^{*} \tau_{i+1}^{*}-r_{i}^{*} \tau_{i}^{*}}{a^{*}}$

the solution of which is $\tau_{i}^{*}=r_{i}^{*}=a^{*} i$. For a Newtonian fluid, the dimensionless shear stress is equal to the r-derivate of the velocity; it is then possible to write:

$\tau_{i}^{*}=\frac{V_{i+1}^{*}-V_{i}^{*}}{a^{*}}$

Combining $\tau_{i}^{*}=r_{i}^{*}$ with Eq. (40), the following equation can be written:

$V_{i+1}^{*}-V_{i}^{*}=a^{*} r_{i}^{*}=a^{* 2} i$

the solution of which is given by Eq (42):

$V_{i}^{*}=\frac{1}{2}\left(a^{* 2} i^{2}-a^{* 2} i\right)+C_{0}$

where $C_{0}$ is a constant computed with the boundary condition $V_{n}{ }^{*}=0$. It is then possible to obtain the following expression:

$V_{i}^{*}=\frac{1}{2}\left(a^{* 2} i^{2}-a^{* 2} i-a^{* 2} n^{2}+a^{* 2} n\right)$

Due to the axisymmetry of the problem, one can note that such a microstructured flow contains a plug flow-like zone of radius $a^{*}$ located around the centre. A parallel can be drawn with the nonlocal model that also possesses a plug flow zone of radius $l_{c}^{*}$ located at the centre of the Poiseuille geometry. The plug flow zone can be attributed to the finite-sized microstructure of the Newtonian fluid.

Eq. (43) can be written substituting $a^{*} i$ by the dimensionless radius $r^{*}$ :

$V_{n}^{*}\left(r^{*}\right)=\frac{1}{2}\left(r^{*} 2-a^{*} r^{*}-1+a^{*}\right)$

It is interesting to note that this solution is also the solution of the second order discrete equations resulting from the combination of Eq. (39) and Eq. (40):

$r_{i}^{*} \frac{V_{i+1}^{*}-2 V_{i}^{*}+V_{i+1}^{*}}{a^{* 2}}+\frac{V_{i}^{*}-V_{i-1}^{*}}{a^{*}}=r_{i}^{*}+r_{i-1}^{*}$

Similarly, the velocity function Eq. (44) is also solution of the following expression derived by the finite-difference approach: 
$r_{i}^{*} \frac{V_{i+1}^{*}-2 V_{i}^{*}+V_{i+1}^{*}}{a^{* 2}}+\frac{V_{i+1}^{*}-V_{i}^{*}}{a^{*}}=2 r_{i}^{*}$

It is also possible to continualise the discrete equation to obtain the nonlocal continualised constitutive law. The discrete relationship given by Eq. (40) can be continualised into:

$\tau^{*}-\frac{a^{*}}{2} \frac{d \tau^{*}}{d r^{*}}=\frac{d V^{*}}{d r^{*}}=r^{*} \quad$ or $\quad \frac{d V^{*}}{d r^{*}}=r^{*}-\frac{a^{*}}{2}$

This equation can be integrated to provide the following velocity profile:

$V^{*}\left(r^{*}\right)=\frac{1}{2}\left(r^{* 2}-a^{*} r^{*}\right)+C_{1}$

where $C_{l}$ is a constant computed with the boundary condition $V^{*}(1)=0$. Finally, the computation of $C_{l}$ shows that the continualised velocity profile is equivalent to the discrete velocity profile given by Eq. (44).

\subsection{Calibration of the nonlocal model with the discrete system}

A mean square error method is used pointing this context to find the value of $l_{c}{ }^{*}$, with respect to $a^{*}$, that gives an optimal fit to the nonlocal velocity profile with respect to the discrete (or continualised) velocity profile. This method has been successfully applied to calibrate the characteristic length $l_{c}{ }^{*}$ value for the application of Eringen nonlocal elasticity for wave propagation [15]. The calibration of $l_{c}{ }^{*}$ consists of minimizing the mean square of the difference between the discrete and the nonlocal velocity profile. This difference can be written as follows:

$\varepsilon_{r r}\left(a^{*}, l_{c}^{*}\right)=\int_{0}^{1}\left[V_{N L}^{*}\left(r^{*}, l_{c}^{*}\right)-V_{D}^{*}\left(r^{*}, a^{*}\right)\right]^{2} d r^{*}$

where $V^{*}{ }_{N L}$ is the nonlocal velocity profile and $V_{D}^{*}$ is the discrete one. This equation can be expanded into:

$\varepsilon_{r r}\left(a^{*}, l_{c}^{*}\right)=\int_{0}^{l_{c}^{*}}\left[\frac{l_{c}^{* 2}-1}{2}-l_{c}^{*^{2}} \ln \left(l_{c}^{*}\right)-\frac{1}{2}\left[r^{* 2}\left(1-\frac{a^{*}}{r^{*}}\right)-1+a^{*}\right]\right]^{2} d r^{*}+\int_{l_{c}^{*}}^{1}\left[-l_{c}^{* 2} \ln \left(r^{*}\right)+\frac{a^{*}\left(r^{*}-1\right)}{2}\right]^{2} d r^{*}$

which leads finally equal to the expression:

$\varepsilon_{r r}\left(a^{*}, l_{c}^{*}\right)=\frac{a^{* 2}}{12}+2 l_{c}^{* 4}-\frac{28 l_{c}^{* 5}}{15}-a^{*} \frac{l_{c}^{* 2}}{24}\left(18-16 l_{c}^{*}+3 l_{c}^{* 2}\right)+\frac{4 l_{c}^{* 5}}{3} \ln \left[l_{c}^{*}\right]$

The minimum difference is obtained when the $a$-derivate of $\varepsilon_{\mathrm{rr}}\left(a^{*}, l_{c}{ }^{*}\right)$ is equal to 0 , then for:

$\frac{\partial \varepsilon_{r r}\left(a^{*}, l_{c}^{*}\right)}{\partial a^{*}}=0 \quad \Rightarrow \quad a^{*}=\frac{l_{c}^{*}\left(18-16 l_{c}^{*}+3 l_{c}^{* 2}\right)}{4}$

The calibration of $l_{c}{ }^{*}$ with respect to $a^{*}$ then gives the following relationship between $l_{c}{ }^{*}$ and the dimensionless thickness $a^{*}$ of the discrete layer:

$a^{*}=\frac{l_{c}^{* 2}\left(18-16 l_{c}^{*}+3 l_{c}^{* 2}\right)}{4}$

which tends to $a^{*}=(9 / 2) l_{c}{ }^{* 2}$ for $l_{c}{ }^{*}<<1$.

The velocity profiles of calibrated nonlocal model and of discrete system for $n=4$ and $n=16$ are plotted on Figure 7. 


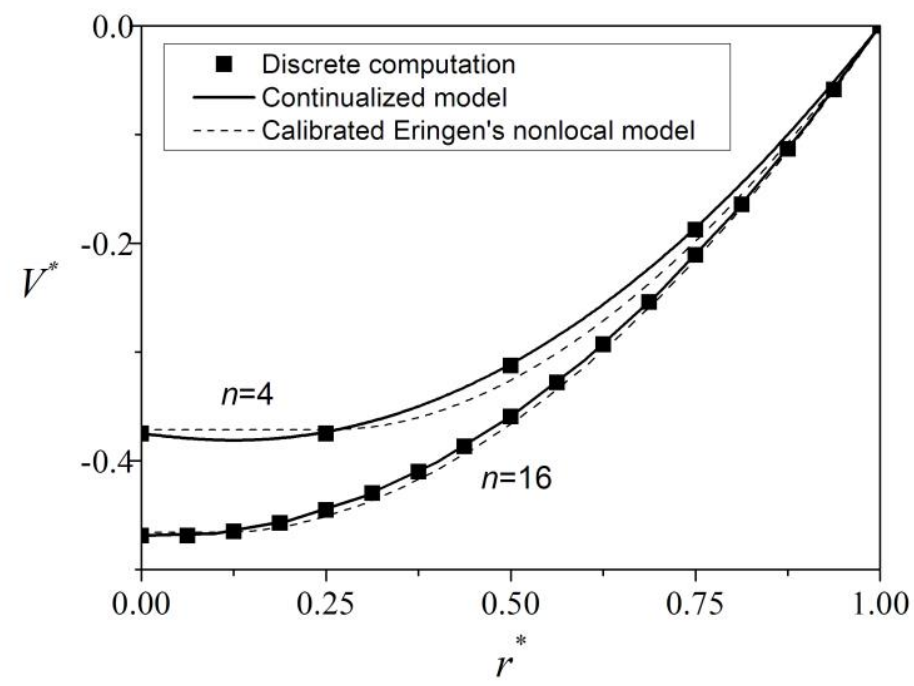

Figure 7: Comparison of velocity profiles obtained with the discrete system, the continualisation of the discrete system and the calibrated nonlocal modelling for $n=4$ and $n=16$.

It appears that the calibrated Eringen's nonlocal model is able to provide a good approximation of the discrete system and can be considered as an efficient engineering model with respect to the flow profile of the discrete system. It is also worth noting that the continualization of the discrete system provides a better model as it resulted in the same velocity profile.

\section{Conclusions}

A nonlocal model has been applied to the rheology of viscoplastic fluids. This model can be classified as an Eringen's type integral-based nonlocal model, which can be also expressed in a differential form. This nonlocal model accounts for some specific microstructural effects characteristic the flow analysis of heterogeneous fluids (such as cement paste, foam, emulsions, granular materials, suspensions, fibred materials...).

Analytical solutions are presented for the Poiseuille axisymmetrical stationary flow. By considering a nonlocal viscous material as a particular case of a nonlocal viscoplastic fluid, it has been shown that a nonlocal viscous fluid may exhibit a pseudo plastic behaviour, this is because a plug flow zone of dimensionless radius $l_{c}{ }^{*}$ appear at the centre of the cylindrical section. In a certain sense, this paper suggests, within a nonlocal mechanics perspective, that it is not necessary to introduce yield stress behaviour in a theoretical model to observe a plug flow zone. This idea was differently presented by Barnes and Walters [16], or more recently by Barnes [17], when discussing the existence of yield stress in viscous fluids.

The comparison of the nonlocal material with a discrete layered material for a Newtonian behaviour also shows the physical basis and potential of the proposed modelling. The introduction of the length scale $l_{c}$ into the nonlocal model, which is related to the nonlocal shear stress, has been calibrated in terms of the microstructure size of the heterogeneous fluid, as recently reported by Challamel et al [18] for non viscous elastic solids. This phenomenological parameter can be inversely adjusted from rheological data.

These results which apply to a simple geometrical configuration need to be generalized for other configurations including some more complex rotational flows.

\section{References :}

[1] J. Goyon, A. Colin, G. Ovarlez, A. Ajdari, L. Bocquet, Spatial cooperativity in soft glassy flows, Nature, 454 (2008) 84-87. 
[2] G.A. Maugin, Generalized continuum mechanics: What do we mean by that, in: Maugin G.A. and Metrikine A.V. Eds, Mechanics of Generalized Continua, One Hundred years after the Cosserats, Springer, in, 2010.

[3] A.C. Eringen, Nonlocal Continuum Field Theories, New-York, 2002.

[4] A.C. Eringen, On nonlocal fluid mechanics, International Journal of Engineering Science, 10 (1972) $561-575$.

[5] B.D. Todd, J.S. Hansen, Nonlocal viscous transport and the effect on fluid stress, Physical Review E, 78 (2008) 051202.

[6] R.M. Puscasu, B.D. Todd, P.J. Daivis, J.S. Hansen, Viscosity kernel of molecular fluids: butane and polymer melts, Physical Review E, 82 (2010) 011801.

[7] W.E. Alley, B.J. Alder, Generalized transport coefficient for hard spheres, Physical Review A, 27 (1983) 31-58.

[8] J. Goyon, A. Colin, L. Bocquet, How does a soft glassy material flow: finite size effects, non local rheology, and flow cooperativity, Soft Matter, 6 (2010) 2668-2678.

[9] G. Ovarlez, S. Cohen-Addad, K. Krishan, J. Goyon, P. Coussot, On the existence of a simple yield stress fluid behavior, Journal of Non-Newtonian Fluid Mechanics, 193 (2013) 68-79.

[10] J. Zhu, J.W. Crawford, J.W. Palfreyman, A general formulation of the reversible stress tensor for a nonlocal fluid, International Journal of Engineering Science, 70 (2013) 124-134.

[11] A.C. Eringen, On differential equations of nonlocal elasticity and solutions of screw dislocation ans surface waves, Journal of Applied Physics, 54 (1983) 4703-4710.

[12] P. Appel, Sur les fonctions hypergéométriques de plusieurs variables, les polynomes d'Hermite et autres fonctions sphériques de l'espace, Mémorial des sciences mathématiques, 3 (1925) 1-75.

[13] F.W.J. Olver, D.W. Lozier, R.F. Boisvert, C.W. Clark, NIST Handbook of Mathematical Functions, Cambridge University Press, New York, 2010.

[14] Wolfram, http://functions.wolfram.com/01.03.03.0001.01, Wolfram Research, Inc. , 2014.

[15] N. Challamel, D. Zorica, T.M. Atanackovic, D.T. Spasic, On the fractional generalization of Eringen's nonlocal elasticity for wave propagation, Comptes Rendus Mécanique, 341 (2013) 298-303.

[16] H.A. Barnes, K. Walters, The yield stress myth?, Rheologica Acta, 24 (1985) 323-326.

[17] H.A. Barnes, The yield stress - a review or ' $\pi \alpha \nu \tau \alpha \rho \varepsilon \mathrm{l}$ ' everything flows?, Journal of NonNewtonian Fluid Mechanics, 81 (1999) 133-178.

[18] N. Challamel, C.M. Wang, I. Elishakoff, Discrete systems behave as nonlocal structural elements: bending, buckling and vibration analysis, European Journal of Mechanics A/solids, 44 (2014) 125135. 